\title{
Elaboração e aplicação de um modelo de visita pós-anestésica em um hospital de ensino de Belém - PA
}

\author{
Elaboration and application of a model of post-anesthesia visit in a teaching hospital of \\ Belém - PA
}

Elaboración y aplicación de un modelo de visita post-anestesia en un hospital universitario de Belém - PA

Williams Barbosa Melo ${ }^{1}$, Anthony Benny da Rocha Balieiro ${ }^{2 *}$, Marcio Lima da Costa ${ }^{2}$, Flavia Moura Gaia Farias ${ }^{2}$, Isabela Sousa Lobato ${ }^{2}$, Jordana de Castro Esteves ${ }^{2}$, Fernando Monteiro Mesquita ${ }^{3}$, Antônio José Malcher Gillet Júnior ${ }^{3}$, Ana Luiza Souza Barbosa ${ }^{3}$, Carla Andréa Avelar Pires².

\section{RESUMO}

Objetivo: Elaborar e validar um modelo de atendimento pós-anestésico em um hospital oncológico de BelémPA. Métodos: É estudo descritivos seccional. Os participantes da pesquisa são residentes e médicos de anestesiologia do Hospital ensino de Belém-PA. O trabalho contou com três fases, sendo a primeira uma submissão de um modelo piloto de visita pós-anestésica para avaliação por anestesiologistas, a fim de receber críticas para a confecção de um modelo sugerido de visita pós-anestésica. Na segunda, o modelo sugerido foi submetido a aplicação e avaliação pelos residentes. Já na terceira fase, o modelo sugerido de visita pós-anestésica foi submetido a avaliação pelos mesmos anestesiologistas, a fim de detectar melhora ou não. Resultados: Avaliações dos anestesiologistas foram boas para ambos os modelos, além de ter ficado evidente que o momento de aplicação de uma visita pós-anestésica deve ser ainda no expediente, deste modo evita-se que o paciente receba alta hospitalar antes da visita pós-anestésica. Conclusão: Ter um modelo de visita pós-anestésica é uma boa forma de melhorar a experiência do paciente com a anestesia e tornar os hospitais mais organizados e padronizados.

Palavras-chave: Anestesia, Período de recuperação da anestesia, Satisfação do paciente.

\begin{abstract}
Objective: To develop and validate a model of post-anesthesia care in an oncologic hospital of Belém-PA. Methods: It is a sectional descriptive study. The participants of the research are residents and anesthesiology doctors of the teaching hospital of Belém-PA. The work consisted of three phases, the first of which was the submission of a pilot model of post-anesthesia visit for evaluation by anesthesiologists in order to receive criticism for the preparation of a suggested model of post-anesthesia visit. In the second phase, the suggested model was submitted to application and evaluation by residents. In the third phase, the suggested model of post-anesthesia visit was submitted to evaluation by the same anesthesiologists in order to detect improvement or not. Results: Evaluation by anesthesiologists was good for both models, and it was evident that the time of application of a post-anesthesia visit should still be during the day, thus avoiding that the patient is discharged from hospital before the post-anesthesia visit. Conclusion: Having a post-anesthesia visit model is a good way to improve the patient's experience with anesthesia and make hospitals more organized and standardized.
\end{abstract}

Key words: Anesthesia, Anesthesia recovery period, Patient satisfaction.

\footnotetext{
${ }^{1}$ Hospital Ophir Loyola (HOL), Belém - PA. *E-mail: anthonybenny1996@outlook.com

2 Universidade Federal do Pará (UFPA), Belém - PA.

${ }^{3}$ Centro Universitário do Estado do Pará (CESUPA), Belém - PA.
} 


\section{RESUMEN}

Objetivo: Desarrollar y validar un modelo de cuidado post-anestesia en un hospital oncológico de Belém-PA. Métodos: Es un estudio descriptivo seccional. Los participantes en la investigación son residentes y médicos anestesistas del hospital universitario de Belém-PA. El trabajo consistió en tres fases, la primera de las cuales fue la presentación de un modelo piloto de visita postanestesia para su evaluación por los anestesiólogos a fin de recibir críticas para la preparación de un modelo sugerido de visita postanestesia. En la segunda fase, el modelo sugerido se sometió a la solicitud y evaluación de los residentes. En la tercera fase, el modelo sugerido de visita posanestesia se sometió a la evaluación de los mismos anestesiólogos a fin de detectar una mejoría o no. Resultados: La evaluación de los anestesiólogos fue buena para ambos modelos, y fue evidente que el momento de aplicación de una visita postanestésica debe seguir siendo durante el día, evitando así que el paciente sea dado de alta del hospital antes de la visita postanestésica. Conclusión: Tener un modelo de visita post-anestesia es una buena manera de mejorar la experiencia del paciente con la anestesia y hacer que los hospitales estén más organizados y estandarizados.

Palabras clave: Anestesia, Periodo de recuperación de la anestesia, Satisfacción del paciente.

\section{INTRODUÇÃO}

Cirurgia é um método de tratamento comum e a principal fonte de receita em muitos hospitais. As respostas fisiológicas ao estresse e ao trauma cirúrgico incluem liberação de substâncias pró inflamatórias, com aumento do estresse oxidativo, consumo de coagulação e acidose metabólica. Algumas respostas metabólicas e fisiológicas à cirurgia causam desequilíbrio nas funções fisiológicas essenciais. Ao mesmo tempo, efeitos retardados de anestésicos e relaxantes musculares enfraquecem a capacidade natural do corpo em restabelecer o estado homeostático e manter sua saúde, resultando em complicações pósoperatórias em unidade de cuidados pós-anestésicos (BAGHERI H, et al., 2018).

A anestesia é um segmento fundamental dos processos assistenciais cirúrgicos e diagnósticos. Assim, é indispensável que o procedimento anestésico seja controlado para garantir resultados assistenciais com excelência. Nesse contexto, pode-se dividir os processos anestésicos da seguinte forma, avaliação préanestésica, procedimento anestésico propriamente dito, analgesia e cuidados pós-operatórios, visita pósanestésica (VPA) e avaliação de satisfação do paciente (SLULLITEL A, 2008).

Com referência a satisfação do paciente, pesquisas indicam que há diferenças entre a percepção de pacientes de países desenvolvidos em comparação aos em desenvolvimento com referência à informação, conscientização, significância e qualidade dos cuidados anestésicos. Variáveis como alfabetização, nível intelectual, restrições econômicas, infraestrutura de saúde e inúmeros outros fatores são responsáveis pela discrepância percebida entre esses pacientes (AUQUIER P, et al., 2005; CALJOUW MA, et al., 2008; CAPUZZO M, et al., 2005; FOSS NB, et al., 2006; SCHIFF JH, et al., 2008).

Acredita-se que a familiarização dos pacientes com o procedimento cirúrgico não só melhora sua consciência e conhecimento, mas também é útil na redução do estresse psicológico da cirurgia. Os pacientes têm a chance de expressar suas preocupações e medos através de educação pré-operatória e um relacionamento interpessoal com foco nas suas necessidades (BAGHERI H, et al., 2018).

O conceito de continuidade do cuidado, isto é, avaliação pré-anestésica, desempenho da anestesia e cuidados pós-operatórios pelo mesmo anestesiologista, é difícil de ser praticado na maioria das configurações de saúde (HEIDEGGER T, et al., 2006). Pesquisas relatam que mesmo em países desenvolvidos há uma crescente deficiência na continuidade de cuidados pelos anestesiologistas (HEIDEGGER T, et al., 2006; SAAL D, et al., 2011).

Segundo Bajwa SJ e Takrouri MS (2013) a prática de VPA por anestesiologistas não é universalmente seguida e, na maioria das instituições, não há protocolos estabelecidos para VPA. O grau de dificuldade é ainda maior, pois não há diretrizes universais estabelecidas sobre os cuidados pós-operatórios pelos anestesiologistas, exceto para um cuidado vigilante na unidade de recuperação pós-anestésica. 
Hoje, com o aumento da procura por uma assistência médica sempre melhor, a relevância VPA foi novamente revivida. Existem pesquisas, as quais observam que as VPA podem aumentar a satisfação do paciente, mas também o prestígio do médico (FINK T, 2017).

Neste contexto, as VPA são necessárias na prática anestésica moderna para sustentar o progresso e os avanços científicos, especialmente nos países em desenvolvimento. Ressalta-se ainda que a garantia da qualidade, a contenção de custos e a segurança do paciente em anestesia podem ser alcançadas pela priorização da preferência do paciente individual. Infelizmente, existem poucos estudos na literatura de anestesia para mostrar como fazer isso corretamente.

Assim, objetiva-se propor um modelo padronizado de atendimento pós-anestésico com validação para aprimorar o cuidado clínico pelo serviço de anestesia hospitalar.

\section{MÉTODOS}

Como a pesquisa é caracterizada como um estudo descritivo seccional e trabalha com seres humanos, foi necessário o uso do Termo de Consentimento Livre e Esclarecido (TCLE). O trabalho foi submetido e aprovado pelo Comitê de Ética em Pesquisa (CEP) do Hospital Ophir Loyola (HOL) de Belém-PA, cujo número do Certificado de Apresentação para Apreciação Ética (CAAE) é 18784719.2.0000.5550 e o número do parecer de aprovação é 3.657.976.

Para elaboração do modelo piloto de VPA proposto se seguiu as orientações das diretrizes práticas para o cuidado pós-anestésico da Sociedade Americana de Anestesiologistas (ASA, 2002). A avaliação da aplicabilidade do modelo foi realizada por profissionais anestesiologistas experientes, com atuação em anestesiologia de mais de 10 anos, que atuam na área de ensino e pesquisa, isto é, staff ou preceptores do serviço. As variáveis relacionadas envolvem características acerca da VPA realizadas por médicos anestesiologistas. $O$ estudo contou das seguintes fases:

1ํ fase: após a elaboração do modelo padronizado de atendimento pós-anestésico, intitulado modelo piloto de VPA, este documento foi entregue aos especialistas experientes (staff do serviços e/ou preceptores) em anestesiologia, para análise quanto à estrutura e conteúdo do modelo elaborado, por meio da avaliação de cada item referente a clareza, relevância, pertinência e abrangência de conteúdo.

Os especialistas responderam se a apresentação gráfica, a orientação sobre a forma de preenchimento e facilidade de leitura, de acordo com a sequência de apresentação dos itens, estavam adequadas no protocolo construído.

Portanto quanto aos itens: a) clareza: avalia se a construção dos itens do modelo proposto, quanto à forma escrita, permite leitura adequada e favorece a compreensão do conteúdo avaliado; b) relevância: indica quanto o item representa o conteúdo que está sendo medido; $c$ ) pertinência: considera se os itens do modelo são adequados e específicos para o conteúdo em avaliação; d) abrangência: mostra se o modelo engloba todos os itens relacionados ao que se deseja mensurar (DEVELLIS RF, 2017).

Cada item do modelo foi pontuado de um a cinco, de acordo com escala tipo Likert sendo: (cinco) concordo totalmente, (quatro) concordo, (três) nem concordo/nem discordo, (dois) discordo, (um) discordo totalmente (DEVELLIS RF, 2017). Nos itens em que houver discordância, o especialista pôde sugerir modificações quanto ao conteúdo proposto.

A escala de Likert é utilizada para medir opiniões, crenças ou atitudes dos respondentes de um questionário ou instrumento, por meio de uma sequência de afirmações, que permite diferentes graus de concordância. A depender do fenômeno de investigação e dos objetivos do investigador, número ímpar ou par de opções de respostas pode acompanhar cada afirmação, sendo que as respostas podem variar de discordo totalmente a concordo totalmente (DEVELLIS RF, 2017).

Essa é uma técnica de classificação bastante comum, a qual integra vários itens que manifestam um ponto de vista sobre um determinado assunto (POLIT DF, et al., 2007). Dessa forma, é possível a obtenção de medidas objetivas, ou seja, é possível quantificar a opinião dos peritos em relação ao manual educativo.

REAS/EJCH | Vol.12(9) | e3905 | DOI: https://doi.org/10.25248/reas.e3905.2020 Página 3 de 9 
Juntamente com o modelo padronizado de atendimento pós-anestésico foi enviado aos juízes uma carta convite. A seleção dos especialistas foi realizada por meio da amostragem bola de neve que consiste na indicação dos sujeitos através de recomendação de outros profissionais, baseados na experiência profissional como educadores e profissionais atuantes na área;

$2^{\underline{a}}$ fase: após o retorno dos especialistas com as sugestões, foi elaborado uma segunda versão do modelo, intitulado modelo sugerido de VPA, o qual foi aplicado aos residentes e médicos anestesiologistas do HOL no intuito de avaliar a aplicabilidade do modelo modificado. Ao fim de desta etapa, eles responderam um questionário sobre a experiência de VPA.

$3^{\text {a }}$ fase: esta fase consistiu na avaliação do modelo sugerido pelos anestesistas mais experientes do serviço, bem como a comparação dessa avaliação com o modelo piloto, a fim de detectar melhora ou piora do modelo sugerido de VPA. Sendo possível, então sugerir ou não este modelo como padronizado para uso no serviço de Anestesiologia do HOL.

Análise dos Dados: As informações da caracterização sobre os dados foram apuradas em banco de dados elaborado no software Microsoft $\Theta^{\circledR}$ Office Excelß 365. Na aplicação da estatística descritiva, foram construídos tabelas e gráficos para apresentação dos resultados e calculadas as medidas de posição como média aritmética e desvio padrão. Um dos principais objetivos da pesquisa em questão visa responder ao seguinte questionamento: é valido o modelo elaborado e padronizado de atendimento pós-anestésico para um serviço de anestesia, de um hospital de ensino de referência em oncologia de Belém-PA?

Para responder a isso, o método de trabalho estatístico analítico, o qual foi empregado para validação de confiabilidade dos questionários foi cálculo do coeficiente alfa de cronbach), além do uso da análise descritiva de medidas centrais e o cálculo de porcentagens (ALMEIDA D, et al., 2010;BUSSAB WO e MORETTIN PA, 2003). Foi aplicado também o teste binomial não-paramétrico, para uma amostra, de forma dicotômica (sim/não), cuja finalidade foi verificar se há diferenças significativas nas proporções da amostra e da população, em cada item avaliado, a fim de verificar se o Modelo proposto foi válido/aceito para o atendimento pós-anestésico (AYRES M, et al., 2007). Para os itens com resposta 100\% de uma única alternativa, seja sim ou não, o teste não foi aplicado, pois não há necessidade diante das respostas obtidas. Somado a isso, o teste $\mathrm{G}$ foi utilizado para avaliar se a os questionários pós-anestésicos, de modelos 1 e 2 , tiveram a mesma concordância ou não, entre os especialistas, ao nível de significância de 5\%.

As variáveis estudadas foram qualitativas ordinais, dicotômicas e quantitativas discretas. As qualitativas ordinais expressam qualidades com características hierárquicas (titulação, escala de Linkert modificada, por exemplo), onde se identifica o crescente e o decrescente nas escalas da variável. As dicotômicas foram variáveis cuja escala apresenta-se em duas alternativas (sim/não, masculino e feminino, por exemplo). As quantitativas discretas foram variáveis numéricas, mas que em sua expressão os valores são inteiros, não admitem decimais por não existirem em intervalos numéricos e sim em um ponto específico (idade, por exemplo). As estatísticas descritiva e analítica foram realizadas no software BioEstat@ 5.3 (AYRES M, et al., 2007). Para a tomada de decisão, foi adotado o nível de significância $\alpha \leq 0,05$ ou $5 \%$ (NETTO AP, 2007).

\section{RESULTADOS}

\section{Fase 1: Validação dos questionários}

Para o instrumento de avaliação dos especialistas o Coeficiente Alfa de Cronbach é de 0,80 para os modelos piloto e sugerido, identificando uma consistência interna quase perfeita nos itens avaliados. No questionário de aplicabilidade do modelo de visitas pós-anestésicas, o coeficiente foi de 0.68 , isto é, uma consistência interna substancial. Ambos os resultados mostram a confiabilidade dos instrumentos utilizados.

Instrumento de avaliação dos especialistas sobre o modelo piloto de VPA: Dois Modelos de instrumentos foram aplicados. Inicialmente foi aplicado o modelo piloto e com as sugestões obtidas pelo tal modelo, o modelo sugerido foi construído e aplicado. Não há diferença estatística entre a concordância dos especialistas para a aplicação dos modelos piloto e sugerido. Então, as informações em tabela abaixo (Tabela 1) são referentes ao modelo piloto.

REAS/EJCH | Vol.12(9) | e3905 | DOI: https://doi.org/10.25248/reas.e3905.2020 Página 4 de 9 
Tabela 1 - Resultado sobre os itens avaliados pelos especialistas. Belém - PA, 2020.

\begin{tabular}{ccc}
\hline Itens Avaliados & Teste G & p-valor \\
\hline Clareza & 0.0976 & 1,0 \\
Relevância & 0.0980 & 1,0 \\
Pertinência & 0.2107 & 1,0 \\
Abrangência & 0.2392 & 1,0 \\
\hline
\end{tabular}

Fonte: Melo WB, et al., 2020.

Estes instrumentos foram aplicados em 8 (oito) especialistas, 50\% do sexo masculino e 50\% feminino com idade média de 54 anos $(54 \pm 12,8)$, variando de 38 a 80 anos, sendo a idade de 46 anos a média. Todos os especialistas têm um tempo de atuação profissional acima de 10 anos. O modelo piloto foi aplicado e os itens como clareza, relevância, pertinência e abrangência foram bem avaliados (Tabela 2), onde todos os especialistas concordam ou concordam completamente com o uso do questionário Modelo de visitas pósanestésicas.

Apenas no item abrangência um especialista (12\%) nem concorda e nem discorda com o uso do questionário. No item relevância $68 \%$ dos especialistas concordam completamente com o uso e quanto à pertinência $63 \%$ concordam. $50 \%$ dos especialistas são concordantes ou completamente concordantes com relação à clareza do questionário e 50\% concordam que ele tenha a abrangência desejada.

Tabela 2 - Avaliação dos modelos aplicados no atendimento pós-anestésicos de VPA. Sendo o modelo 1 (piloto) e modelo 2 (sugerido). Belém - PA, 2020.

\begin{tabular}{ccccc}
\hline \multicolumn{7}{c}{ Clareza Relevância } & Pertinência Abrangência \\
\hline Discordo totalmente & & & & \\
\hline Discordo & & & & \\
\hline Nem concordo/nem discordo & & & & $12 \%$ \\
\hline Concordo & $50 \%$ & $37 \%$ & $63 \%$ & $50 \%$ \\
\hline Concordo totalmente & $50 \%$ & 63 & $37 \%$ & $38 \%$ \\
\hline
\end{tabular}

Fonte: Melo WB, et al., 2020.

Ao aplicar o modelo sugerido, os resultados mostraram melhorias em todos os itens avaliados em comparação ao modelo piloto. Nos itens relevância e pertinência, $75 \%$ dos especialistas concordam completamente com este modelo e $63 \%$ concordam que ele apresenta clareza e abrangência.

\section{Fase 2: Questionário de aplicabilidade do modelo sugerido de VPA}

Nessa fase, o questionário foi aplicado durante o turno de estágio na residência médica por $83 \%$ dos especialistas, enquanto $17 \%$ preferiram aplicar o questionário após o turno (Gráfico 1).

Entre os 30 participantes selecionados, apenas em 2 (dois) participantes o questionário não foi possível ser aplicado. Nos 28 (93\%) restantes, não houve dificuldade de execução da pesquisa, sendo esta proporção significativa $(p<0,0001)$. Desta forma a amostra passou a ser de $28(100 \%)$ participantes em que 0 questionário foi aplicado.

Em $100 \%$ dos participantes o questionário ajudou a guiar o especialista e melhorou a relação médicopaciente. Em $88 \%$ dos participantes houve a afirmação de aplicação do questionário não detectou complicações contra $12 \%$ que conseguiu detectar alguma forma de aplicação. Além disso, ficou evidente que poucos tiveram dificuldade aplicar o modelo, isto é, apenas $7,15 \%$, enquanto $92,85 \%$ não referiram tal dificuldade (Tabela 3). 
Gráfico 1 - Respostas médicos e residentes de anestesiologia do HOL sobre o instrumento de avaliação. Belém - PA, 2020.

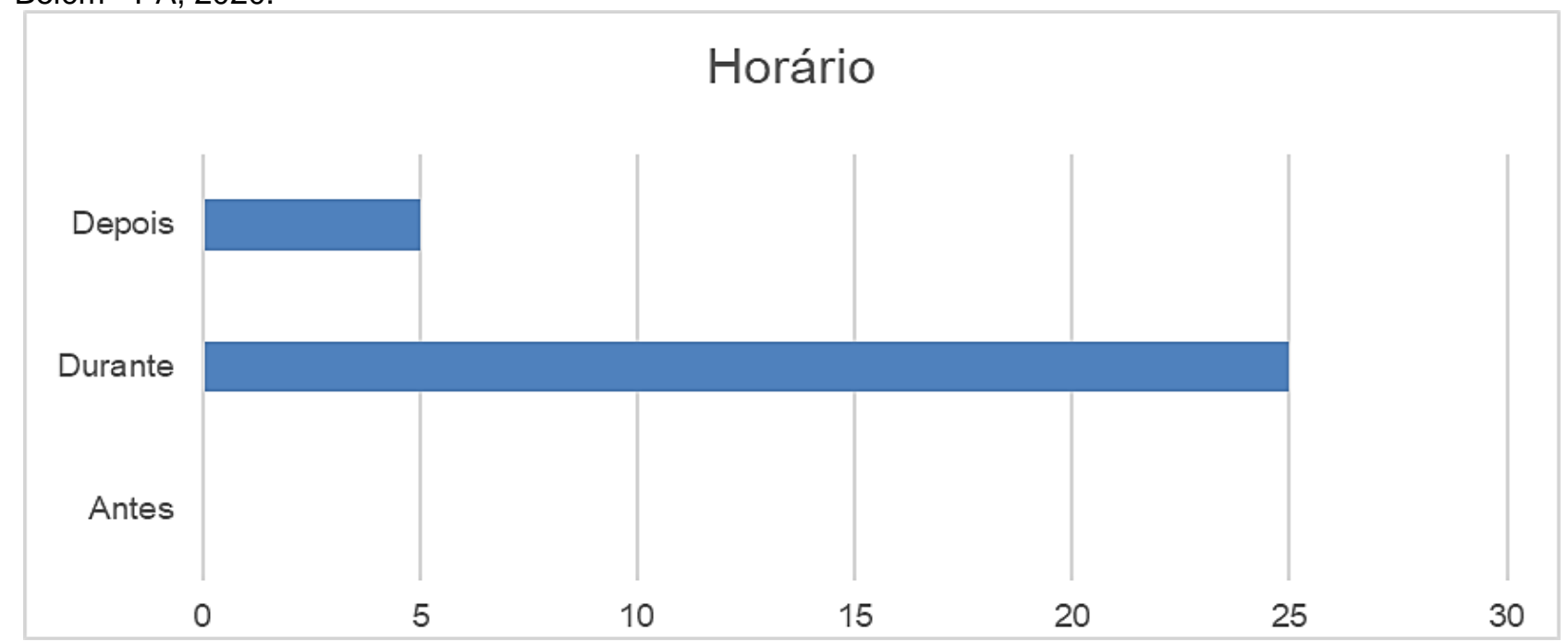

Fonte: Melo WB, et al., 2020.

Tabela 3 - Respostas médicos e residentes de anestesiologia do HOL sobre o instrumento de avaliação. Belém - PA, 2020.

\begin{tabular}{ccc}
\hline Alternativas & Sim & Não \\
\hline Dificuldade para aplicação & $7,15 \%$ & $92,85 \%$ \\
Ajudou a guiá-lo & $100 \%$ & 0 \\
Detectadas complicações & $12 \%$ & $88 \%$ \\
Melhora relação médico-paciente & $100 \%$ & 0 \\
\hline
\end{tabular}

Fonte: Melo WB, et al., 2020.

\section{Fase 3: Modelos de VPA}

Conforme as fases da pesquisa foram sendo feitas, surgiram resultados sobre novos modelos de VPA, pois os anestesiologistas experientes recomendaram mudanças (Quadro 1), o que era uma das intenções desta fase, então foi elaborado o modelo sugerido de VPA como resultado, o qual já acrescentou as modificações sugeridas pelos mais experientes médicos. Com isso, esse segundo modelo foi indicado para aplicação e avaliação dos residentes, o que gerou resultados já descrito no parágrafo anterior na fase 2 .

Além disso, o modelo sugerido também foi submetido a avaliação dos anestesistas mais experientes da mesma forma como o modelo piloto (Tabela 4), inclusive com o mesmo questionário, a fim de poder realizar comparação entre as avaliações partir disso, então foi feita uma comparação com o objetivo da saber se houve melhora ou piora no modelo sugerido, para que este possa ser indicado para uso no HOL. Ressaltase que não houve diferenças estatísticas entre o modelo piloto e sugerido. Entretanto, ao nível descritivo, identifica-se um aumento nas proporções para o nível de 'concordo completamente', quando nos referimos ao modelo sugerido, o que houve melhorias.

Para fins de comparação entre os modelos, o que ficou evidente de diferenças foram maior marcação em algum item de avalição ou até mesmo ausência de marcação em algum item. Dessa forma, no modelo sugerido, a respeito do item abrangência, nenhum participante marcou a opção "nem concorda nem discorda" com o uso do questionário como ocorreu no modelo piloto. Além disso, no item relevância, houve um aumento na marcação desse item, passando de $68 \%$ para $75 \%$ no modelo sugerido. Quanto ao item sobre a pertinência, obteve-se que o valor de $63 \%$ passou a ser $75 \%$ que concordam completamente. Sobre a clareza, o valor de $50 \%$ dos especialistas os quais eram concordantes totalmente passou a ser $67 \%$. A respeito da abrangência desejada, os $38 \%$ que concordavam totalmente passaram a ser sendo $68 \%$. 
Quadro 1 - Alterações realizadas, de acordo com as recomendações dos anestesiologistas mais experientes, para criação do modelo sugerido de VPA a ser aplicado aos médicos e residentes de Anestesiologia do HOL. Belém - PA, 2020.

\begin{tabular}{|l|}
\hline Alterações realizadas nos itens do modelo \\
\hline O questionário ficou mais objetivo, incluindo agora o registro do paciente na parte de identificação inicial. \\
\hline $\begin{array}{l}\text { O termo avaliação básico foi alterado para exame físico, direcionado para os principais sistemas } \\
\text { orgânicos que se alteram durante uma anestesia. }\end{array}$ \\
\hline $\begin{array}{l}\text { Na avaliação respiratória foi removido saturação de oxigênio e perviedade da via aérea e incluído } \\
\text { dispneia e suporte ventilatório. }\end{array}$ \\
\hline Na avaliação cardiovascular o quesito pulso foi subdivido em frequência cardíaca e perfusão periférica. \\
\hline Na avaliação neuromuscular foi acrescentado os quesitos fraqueza, paresias e parestesias. \\
\hline No estado mental, os quesitos acordado, sonolento e inconsciente foram acrescentados. \\
\hline $\begin{array}{l}\text { Na avaliação da dor, uma junção da escala facial de dor com o escore de } 0 \text { à } 10 \text { foi colocado para por } \\
\text { sua fácil aplicabilidade e mensuração. }\end{array}$ \\
\hline $\begin{array}{l}\text { A avaliação de náuseas e vomito foi incluído na parte de complicações juntamente com avaliação de } \\
\text { prurido e cefaleia. }\end{array}$ \\
\hline Temperatura e Diurese permaneceram inalteradas. \\
\hline $\begin{array}{l}\text { Drenos e sangramentos foram removidos por estar mais ligada ao procedimento cirúrgico que anestésico, } \\
\text { sendo o mesmo de responsabilidade da equipe cirúrgica. }\end{array}$ \\
\hline Satisfação do paciente foi adicionado para avaliação e críticas do mesmo sobre o ato anestésico. \\
\hline
\end{tabular}
Fonte: Melo WB, et al., 2020.

Tabela 4 - Respostas dos anestesiologistas mais experientes sobre a avaliação do modelo sugerido de VPA. Belém - PA, 2020.

\begin{tabular}{|c|c|c|c|c|c|}
\hline & Clareza & Relevância & Pertinência & Abrangência & \\
\hline Discordo totalmente & & & & & \multirow{5}{*}{$\begin{array}{l}\text { Modelo } \\
\text { sugerido }\end{array}$} \\
\hline Discordo & & & & & \\
\hline \multicolumn{5}{|c|}{ Nem concordo/nem discordo } & \\
\hline Concordo & $37 \%$ & $25 \%$ & $25 \%$ & $37 \%$ & \\
\hline Concordo totalmente & $63 \%$ & $75 \%$ & $75 \%$ & $63 \%$ & \\
\hline
\end{tabular}

Fonte: Melo WB, et al., 2020.

\section{DISCUSSÃO}

Os principais resultados desse trabalho demonstraram que a maioria dos especialistas experientes "concordaram" ou "concordaram totalmente" nos itens do modelo piloto de VPA quanto clareza, relevância, pertinência e abrangência. Isso demonstra como o conhecimento e contribuição desses profissionais acerca da construção do primeiro modelo piloto estava em consonância com as práticas em anestesiologia sobre a VPA, o que foi fundamental para a criação do segundo modelo: o sugerido. Ademais, embora com a exclusão desses 2 indivíduos nos testes, com os 28 participantes restantes se nota que não houve dificuldade de aplicação dos questionários.

Nesse sentido, 64\% dos participantes afirmaram que a aplicação do questionário não detectou complicações contra $38 \%$ que conseguiu detectar. Além disso, a diferença entre as proporções amostradas e as populacionais $(50 \%$ para SIM e $50 \%$ para NÃO) não foram significativas $(p=0,1367)$. Assim, quanto a este item, é preciso identificar como melhorar a detecção de complicações no modelo de VPA, considerando que a proporção de não detecção foi descritivamente maior.

Somado a isso, a avaliação aos médicos e residentes em Anestesiologia, nota-se que em apenas 2 (dois) participantes, o questionário não foi possível ser aplicado, uma vez que tentaram realizar a aplicação após o expediente, assim fica a sugestão de que a VPA seja realizada ainda durante o expediente, preferencialmente, pela manhã. 
Ressalta-se que o presente estudo demonstrou que há significância nos instrumentos de avaliação sobre os questionários de VPA tanto na fase 1 da pesquisa como na fase 2 , ou seja, tiveram sucesso na comprovação de significância estatística de acordo com o coeficiente Alfa de Cronbach ( $\alpha$ ). De acordo com Gonçalves L (2017), tal coeficiente é uma medida muito utilizada de confiabilidade. Em essência, o Alfa de Cronbach quantifica a consistência interna dos itens que formam um construto, isto é, se os itens estão "na mesma direção". O Alfa de Cronbach é uma medida que varia de 0 a 1 , onde valores mais altos indicam maior consistência interna do constructo. Dessa forma, como na fase 1 e 2 tiveram valores menores que 1, podese afirmar que os instrumentos de avaliação usados são validados e tem significância.

Diante do exposto, levando em consideração a dificuldade de aplicação da VPA após o expediente, devese deixar claro que a realização das VPA é mais comum durante o expediente, bem como é mais preconizada nesse período (SILVEIRA LZ, 2017). Conforme Fink T et al. (2017), muitos anestesistas afirmam que a falta de tempo para fazer as VPA é o motivo de não ser realizada, pois a maioria desses médicos alegam que os pacientes já recebem alta hospitalar quando feita após o expediente, o que também reforça a necessidade de ter uma VPA o quanto antes após o procedimento cirúrgico.

Além disso, ficou evidente que o modelo sugerido de VPA teve uma boa aceitação e avaliação, porque $100 \%$ dos participantes respondeu que foi uma ajuda sobre guiar o especialista e melhorar a relação médicopaciente. Isso vai de acordo com as pesquisas de Silveira LZ (2017), a qual foi realizada no mesmo hospital ( $\mathrm{HOL})$, onde todos os residentes de anestesiologia afirmaram que as visitas pós-anestésicas, realmente, trazer bons resultados sobre a redução da incidência de complicações.

Ademais, é comum vários ou todos os anestesiologistas considerarem a VPA como algo bem relevante, pois segundo Silveira LZ (2017), em seus estudos sobre a importância da VPA, nenhum médico anestesista considera a VPA irrelevante, mas sim a considera como importante na maioria ou todas as ocasiões de sua aplicação. Dessa forma, Fink $T$ et al. (2017) demonstrou também que a maioria dos anestesiologistas acreditam que a VPA melhora a qualidade da prática anestésica, bem como eles admitem que VPA quando feita de forma rotineira devem reduzir as complicações pós-operatórias. Nesse sentido, sabemos que as VPA pouco são realizadas nos hospitais, pois já há estudos os quais evidenciam que uma minoria dos pacientes recebe VPA, sendo que a menor porcentagem se encontra na categoria de especialistas (staff) (SILVEIRA LZ, 2017; FINK T, et al., 2017).

Com base na significância desses instrumentos de avaliações sobre um modelo de VPA, fica evidente que implementar um modelo como o segundo ou dito sugerido, dessa pesquisa, é uma maneira de considerar, mas também ressaltar a grande apreciação, significativa das VPA e as potenciais consequências médicolegais quando é negligenciada. Assim, parece desejável implantar melhorias organizacionais para os cuidados pós-anestésica em um hospital (SILVEIRA LZ, 2017).

Logo, devemos levar em consideração que a medicina está cada vez mais competitiva e exigindo não só mais conhecimento dos médicos, mas também melhorias do ponto de vista de estrutura e organização dos serviços de anestesiologia, ou seja, ter um modelo de VPA padronizado, bem como realizado por anestesistas de maneira regular durante o expediente pode ser uma das formas de melhorar a organização de um serviço, mas também a experiência dos pacientes com a anestesia. Afinal, é razoável compreender que as VPA quando seguem um padrão de aplicação devem não apenas permitir o tratamento de maneira precoce, mas também diferenciada de complicações, o que pode reduzir os custos hospitalares, a mortalidade e permanência da internação do paciente (BAJWA SJ e TAKROURI MS, 2013).

Com base nisso, sabemos que a padronização de uma VPA e sua aplicação de forma rotineira de sua importância também a respeito do que concerne a satisfação do paciente. Segundo Bauer $M$ et al. (2001), a satisfação do paciente com a experiência da anestesia pode ser melhorada pelas VPA, principalmente, quando feito presencialmente. Nesse sentido, Capuzzo M et al. (2005). Evidenciou que quando mais de duas VPA são feitas após a cirurgia, há um aumento significativo acerca da satisfação do paciente. Tudo isso demonstra o quanto a padronização da VPA pode trazer benefícios ao paciente. No entanto, para chegar nessa aplicação de rotina de VPA de maneira padronizada, Fink $T$ et al. (2007) ressalta a necessidade da implantação de um serviço voltado para realizar as VPA, sendo uma forma de aumentar a quantidade de pacientes visitados logo após as cirurgias.

REAS/EJCH | Vol.12(9) | e3905 | DOI: https://doi.org/10.25248/reas.e3905.2020 Página 8 de 9 


\section{CONCLUSÃO}

Por meio desse estudo que ficou evidente que no HOL as VPA são pouco realizadas no serviço de anestesiologia, entretanto todos reconhecem que é muito importante que se faça. Dessa forma, levando em consideração o grande valor dessas visitas pelos anestesiologistas atualmente, bem como a ocorrência de eventuais complicações pós-operatórias detectadas durante essas as VPA, é necessário ter melhorias organizacionais para pós-anestésica nos hospitais, sendo um modelo de VPA uma alternativa para isso, tendo essa presente pesquisa o seu principal objetivo, este sendo feito com significância estatística para fins de relevância. Logo, o modelo sugerido não é apenas um guia para realizar um VPA, mas também um instrumento de pesquisa sobre o tema, uma maneira de autoavaliação para os próprios anestesiologistas, mas também uma forma de evitar complicações, permanência hospitalar, mortalidade e os custos.

\section{AGRADECIMENTOS E FINANCIAMENTO}

Fica o agradecimento a toda equipe de médicos e residentes de Anestesiologia do Serviço do HOL, bem como a própria instituição por permitir e colaborar com essa pesquisa.

\section{REFERÊNCIAS}

1. ALMEIDA D, et al. Aplicação do coeficiente Alpha de Cronbach nos resultados de um questionário para avaliação de desempenho da saúde pública. In: XXX Encontro de Engenharia de Produção. 2010, São Carlos, SP.

2. AMERICAN SOCIETY OF ANESTHESIOLOGISTS (ASA). Practice Guidelines for Postanesthetic Care. Anesthesiology, 2002; 96(3):742-52

3. AUQUIER P, et al. Development and validation of a perioperative satisfaction questionnaire. Anesthesiology, 2005; 102(6):1116-23.

4. AYRES M, et al. Bioestat 5.0. Aplicações estatísticas nas áreas de ciências biológicas e médicas. 2007.

5. BAGHERI H, et al. Effect of Preoperative Visitation by Operating Room Staff on Preoperative Anxiety in Patients Receiving Elective Hernia Surgery. J Perianesth Nurs,2019; 34 (2):272-280.

6. BAJWA SJ, TAKROURI MS. Post-operative anesthesia rounds: Need of the hour. Anesth Essays Res, 2013; 7 (3): 291-293.

7. BAUER M, et al. Measuring patient satis-faction with anaesthesia: perioperative questionnaire versusstandardised face-to-face interview. Acta Anaesthesiol Scand.2001;45(1): 65-72.

8. BUSSAB WO, MORETTIN PA. Estatística Básica. São Paulo: Editora Saraiva, $5^{\mathrm{a}}$ ed. 2003; 526p.

9. CALJOUW MA, et al. Patient's satisfaction with perioperative care: Development, validation, and application of a questionnaire. Br J Anaesth, 2008; 100 (5): 637-44.

10. CAPUZZO M, et al. Emotional and interpersonal factors are most important for patient satisfaction with anaesthesia. Acta Anaesthesiol Scand, 2005; 49 (6):735-42.

11. DEVELLIS RF. Scale development. Theory and applications. $4^{a}$ ed. Los Angeles: Sage, 2017. p. 106-51

12. FINK T, et al. A prática de visitas pós-anestésicas - estudo de um questionário. Rev Bras Anestesiol, 2017; 67(6): 571577.

13. FOSS NB, et al. Post-operative rounds by anaesthesiologists after hip fracture surgery: A pilot study. Acta Anaesthesiol Scand, 2006; 50 (4): 437-42.

14. GONÇALVES L. Validação de Instrumentos de Pesquisa. ABG Consultória, 2017. Disponível em: https://www.abgconsultoria.com.br/blog/importancia-da-validacao-de-instrumentos/. Acesso em: 21 de dez. de 2019.

15. HEIDEGGER T, et al. Patient satisfaction with anaesthesia care: What is patient satisfaction, how should it be measured, and what is the evidence for assuring high patient satisfaction? Best Pract Res Clin Anaesthesiol, 2006;20(2): 331-46.

16. NETTO AP. Como Interpretar a Significância Estatística. Diabetes, 2007. Disponível em:https://www.diabetes.org.br/publico/colunistas/20-dr-augusto-pimazoni-netto/125-como-interpretar-a-significanciaestatistica. Acesso em: 21 de dez. de 2019

17. POLIT DF, et al. Is the CVI an Acceptable Indicator of Content Validity? Appraisal and Recommendations. Res Nurs Health. [Internet]. v. 30, n. 4, p. 459-67, 2007. Disponível em: http://onlinelibrary.wiley.com/doi/10.1002/nur.20199/full. Acesso em: 22 jun. 2019.

18. SAAL D, et al. Does a postoperative visit increase patient satisfaction with anaesthesia care? $\mathrm{Br} J$ Anaesth, 2011; 107 (5): 703-9.

19. SCHIFF JH, et al. The Heidelberg peri-anaesthetic questionnaire-development of a new refined psychometric questionnaire. Anaesthesia, 2008; 63(1):1096-104.

20. SILVEIRA LZ. Perfil das visitas pós-anestésicas em um hospital oncológico em Belém-Pa. Trabalho de conclusão de residência médica (Residência em Anestesiologia) - Serviço de Anestesiologia, Hospital Ophir Loyola, Belém, 2017; $41 \mathrm{p}$.

21. SLULLITEL A. Gestão da qualidade em anestesiologia. Prática hospitalar, 2008; 58(1): 93-96.

22. SYME P, CRAVEN R. Recovery and postanaesthetic care. Anaesthesia \& Intensive Care Medicine, 2009; 10 (12): $576-579$. 